Supporting Information

\title{
Hierarchical $\mathrm{Ti}_{3} \mathrm{C}_{2} \mathrm{~T}_{\mathrm{x}}$ MXene/Ni Chain/ZnO Array
}

Hybrid Nanostructures on Cotton Fabric for Durable

\section{Self-Cleaning and Enhanced Microwave Absorption}

\author{
Shijun Wang ${ }^{1}$, Diansen Li $^{1,2 *}$, Yue Zhou ${ }^{1}$, Lei Jiang ${ }^{1}$ \\ ${ }^{1}$ Key Laboratory of Bio-Inspired Smart Interfacial Science and Technology, Ministry of \\ Education, School of Chemistry, Beihang University, Beijing 100191, China
}

${ }^{2}$ Beijing Advanced Innovation Center for Biomedical Engineering, Beihang University, Beijing 100191, China

* Corresponding author: E-mail: lidiansen@buaa.edu.cn 

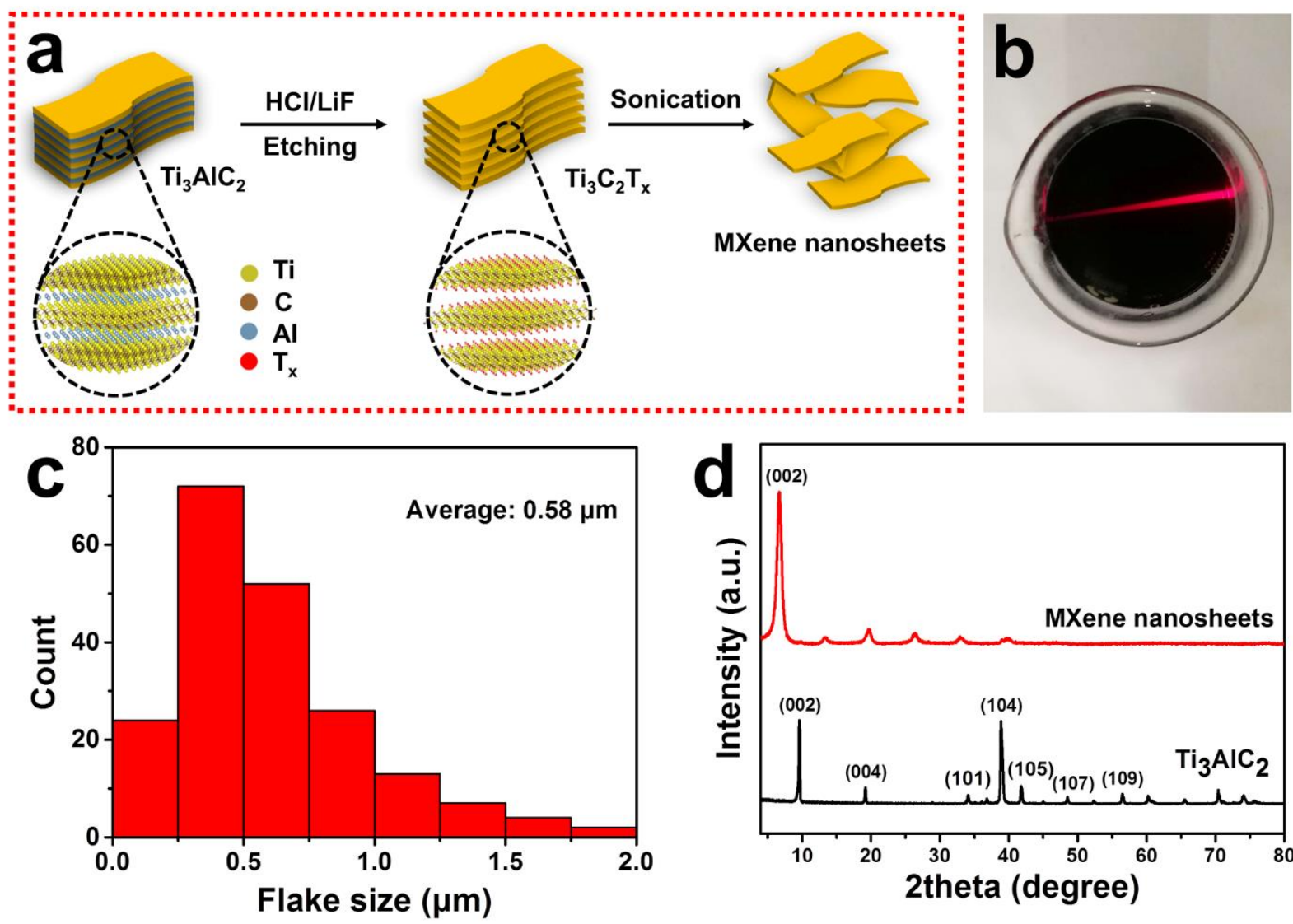

MXene nanosheets

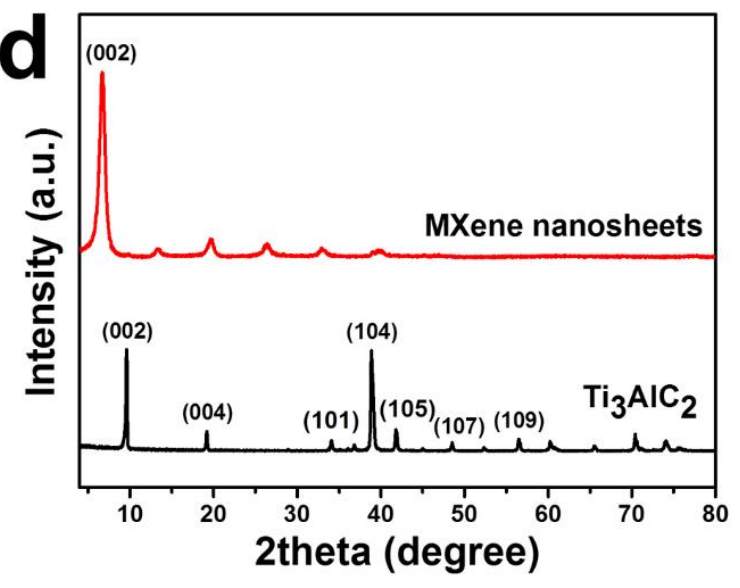

Figure S1. (a) Schematic preparation process of MXene nanosheets. (b) Tyndall scattering effect of MXene nanosheet solution. (c) Size distribution of MXene nanosheets measured from SEM over 200 sheets. (d) XRD patterns of $\mathrm{Ti}_{3} \mathrm{AlC}_{2}$ and MXene nanosheets. 

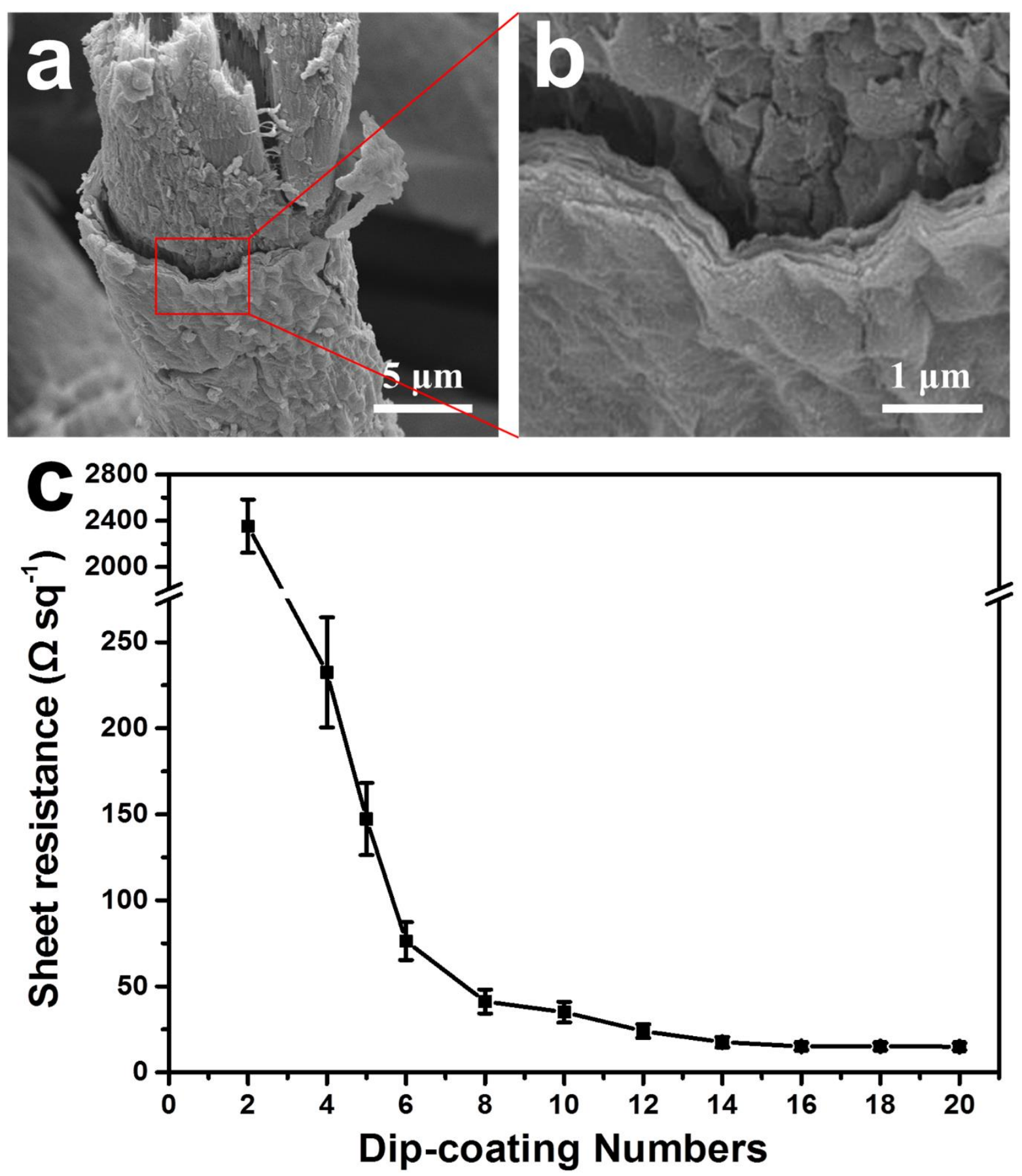

Figure S2. (a,b) Cross-sectional SEM images of CF-M fabric. (c) Sheet resistances of CF-M fabrics with different dip-coating numbers. 

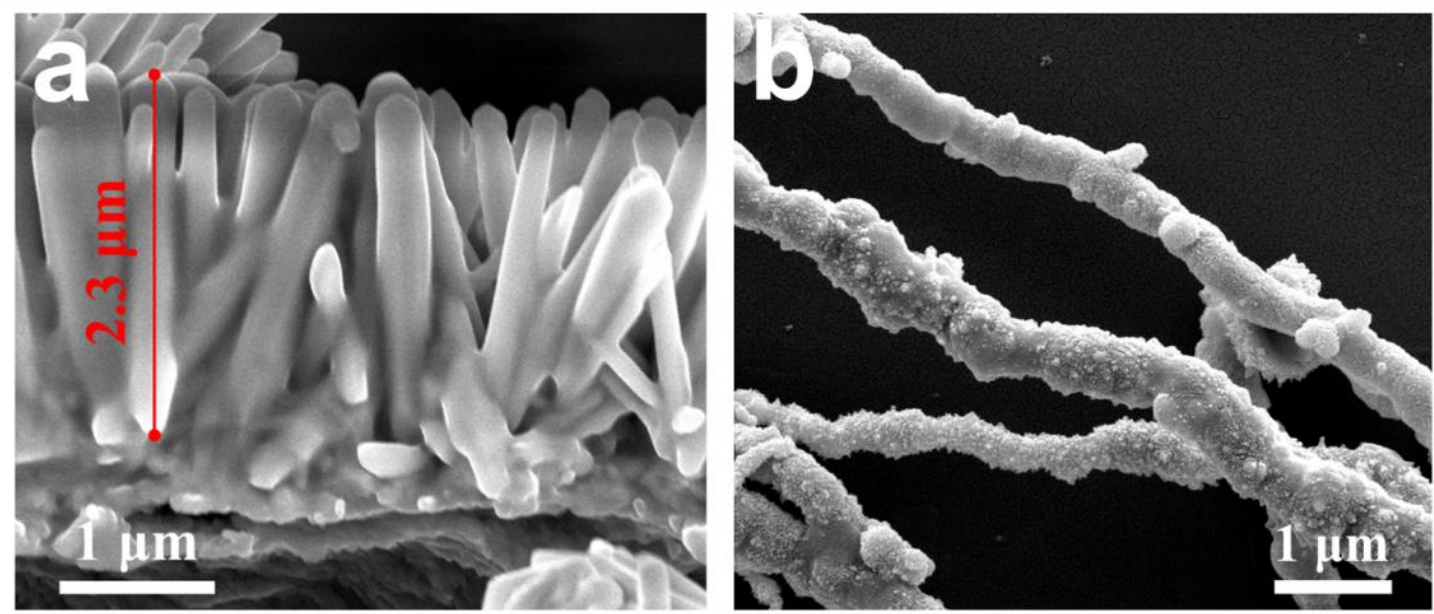

Figure S3. (a) Cross-sectional SEM image of CF-MNZ fabric. (b) SEM image of Ni chains.
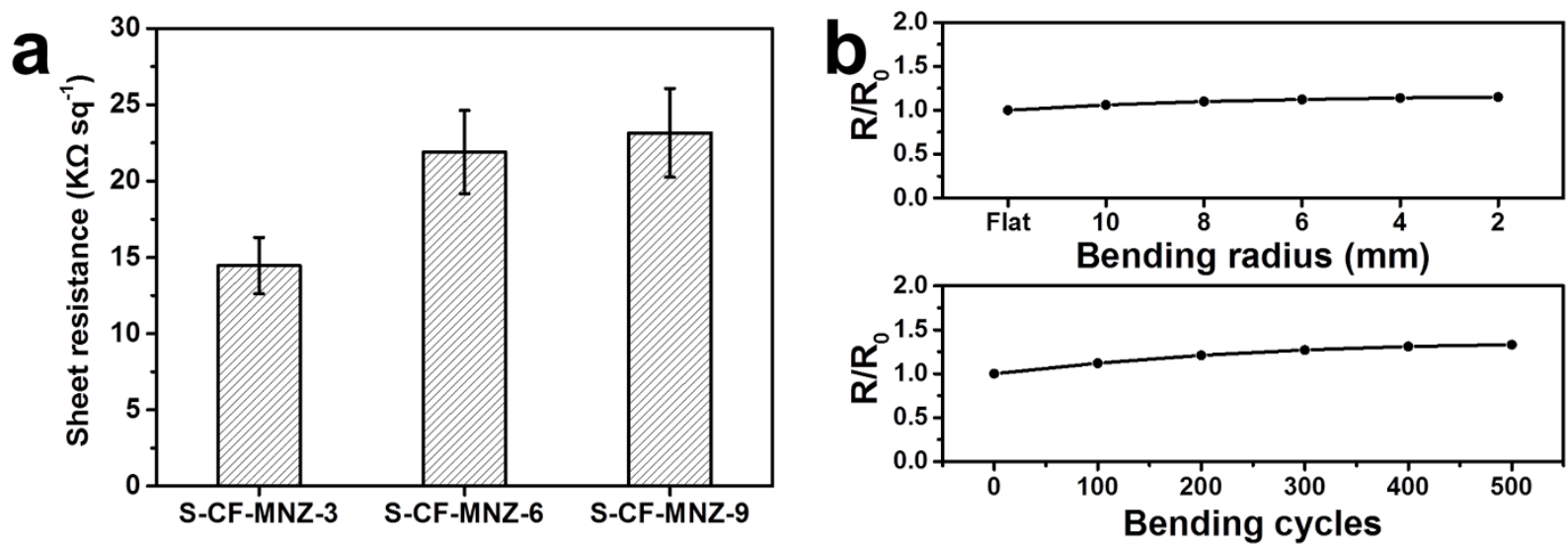

Figure S4. (a) Sheet resistances of S-CF-MNZ fabrics. (b) Relative resistance changes of S-CFMNZ-9 fabrics as a function of bending radius and bending cycles.
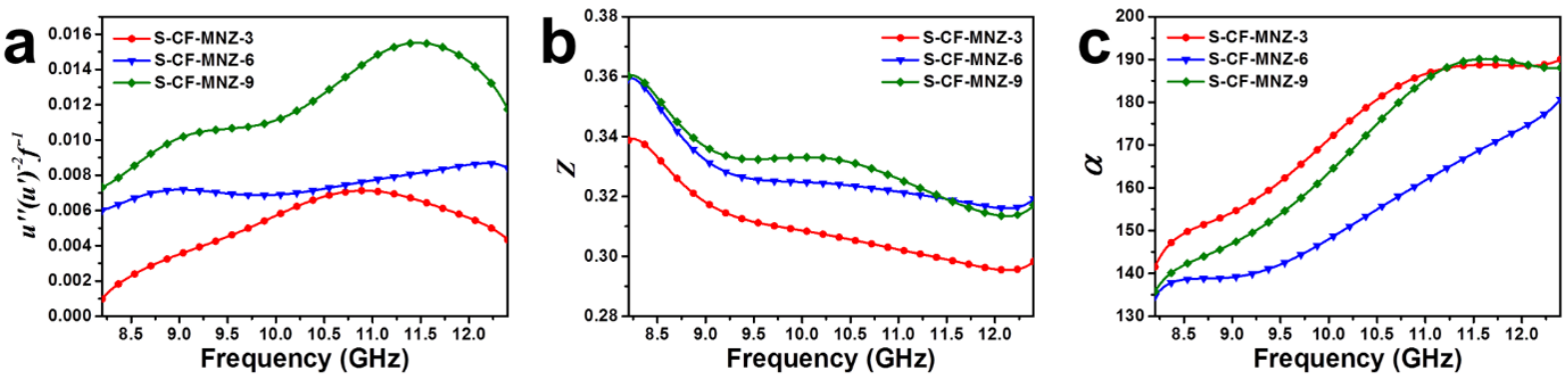
Figure S5. (a) $\mu^{\prime \prime}\left(\mu^{\prime}\right)^{-2} f^{-1}$, (b) Z and (c) $\alpha$ values of S-CF-MNZ-3, S-CF-MNZ-6 and S-CF-MNZ-9 fabrics.

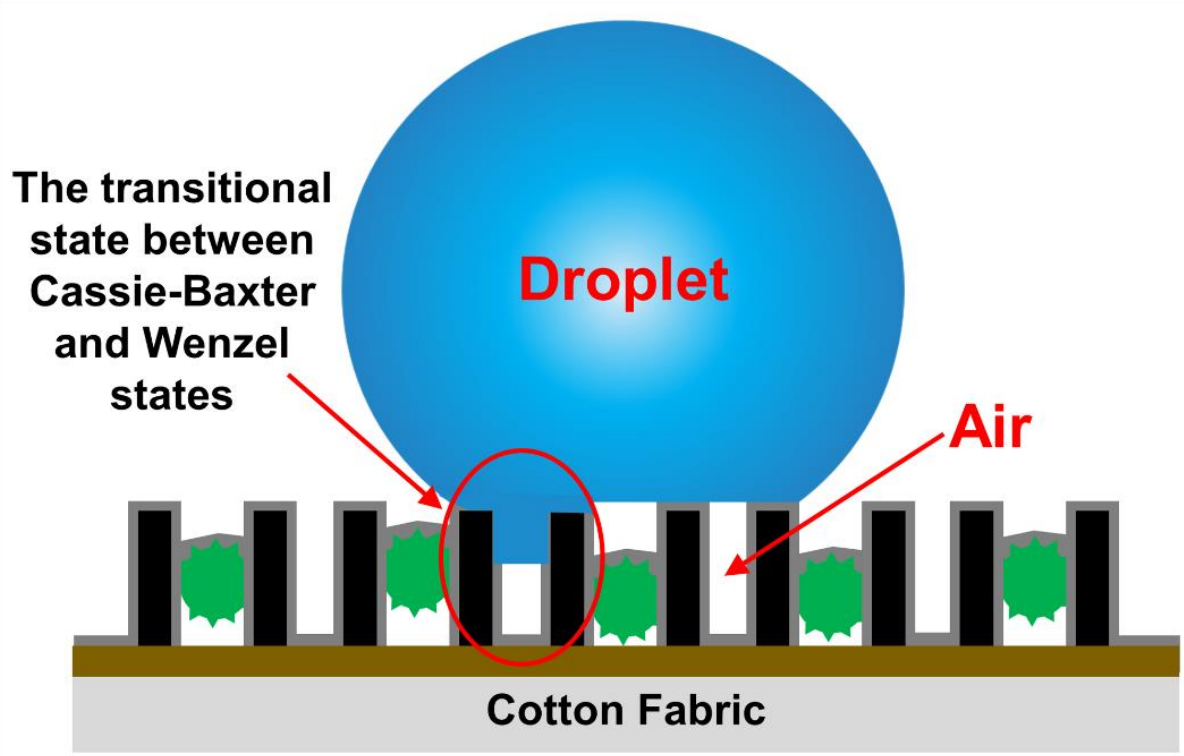

Figure S6. Schematic illustration of liquid droplet on S-CF-MNZ-9 fabric surface after sandpaper abrasion test.
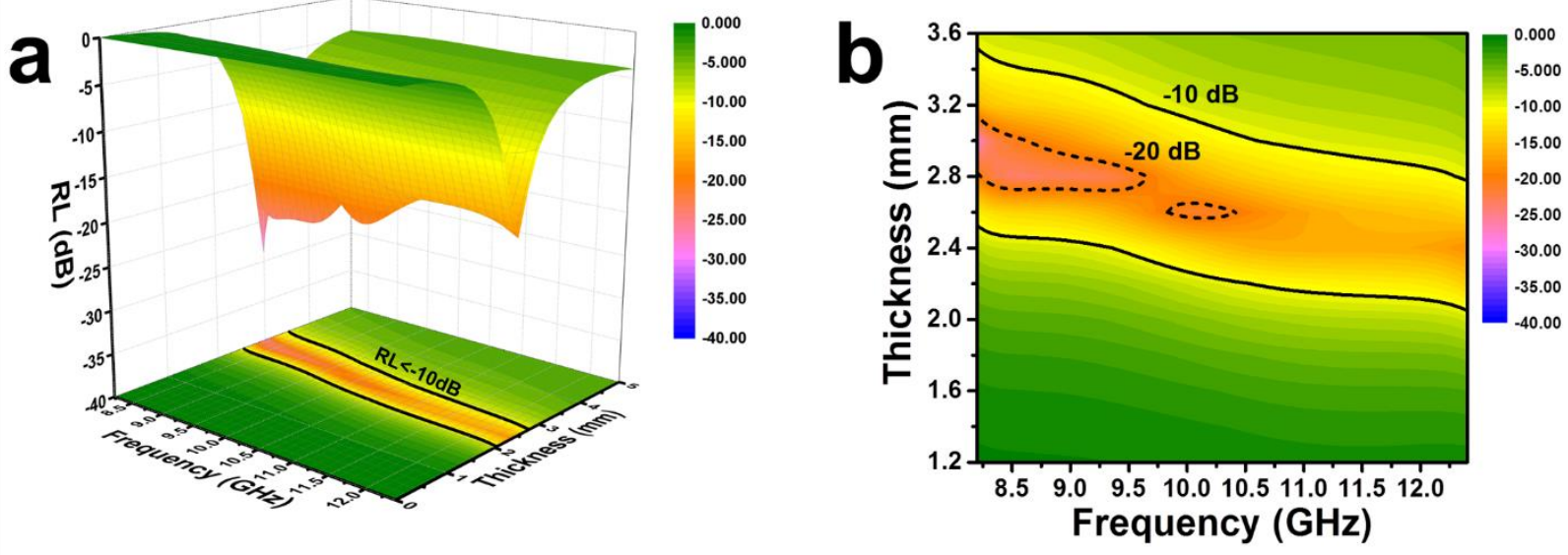

Figure S7. (a,b) RL values of S-CF-MNZ-9 fabrics after 20 times of the abrasion cycles. 


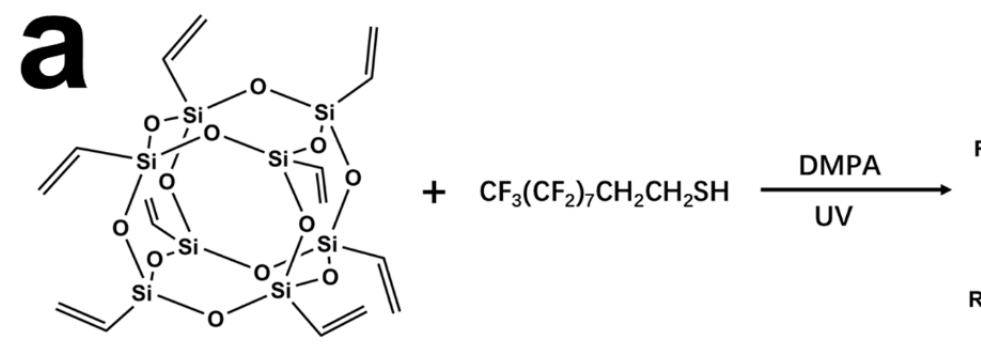

(viny-POSS)

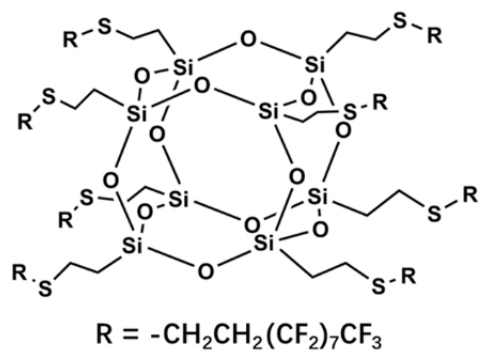

(F-POSS)

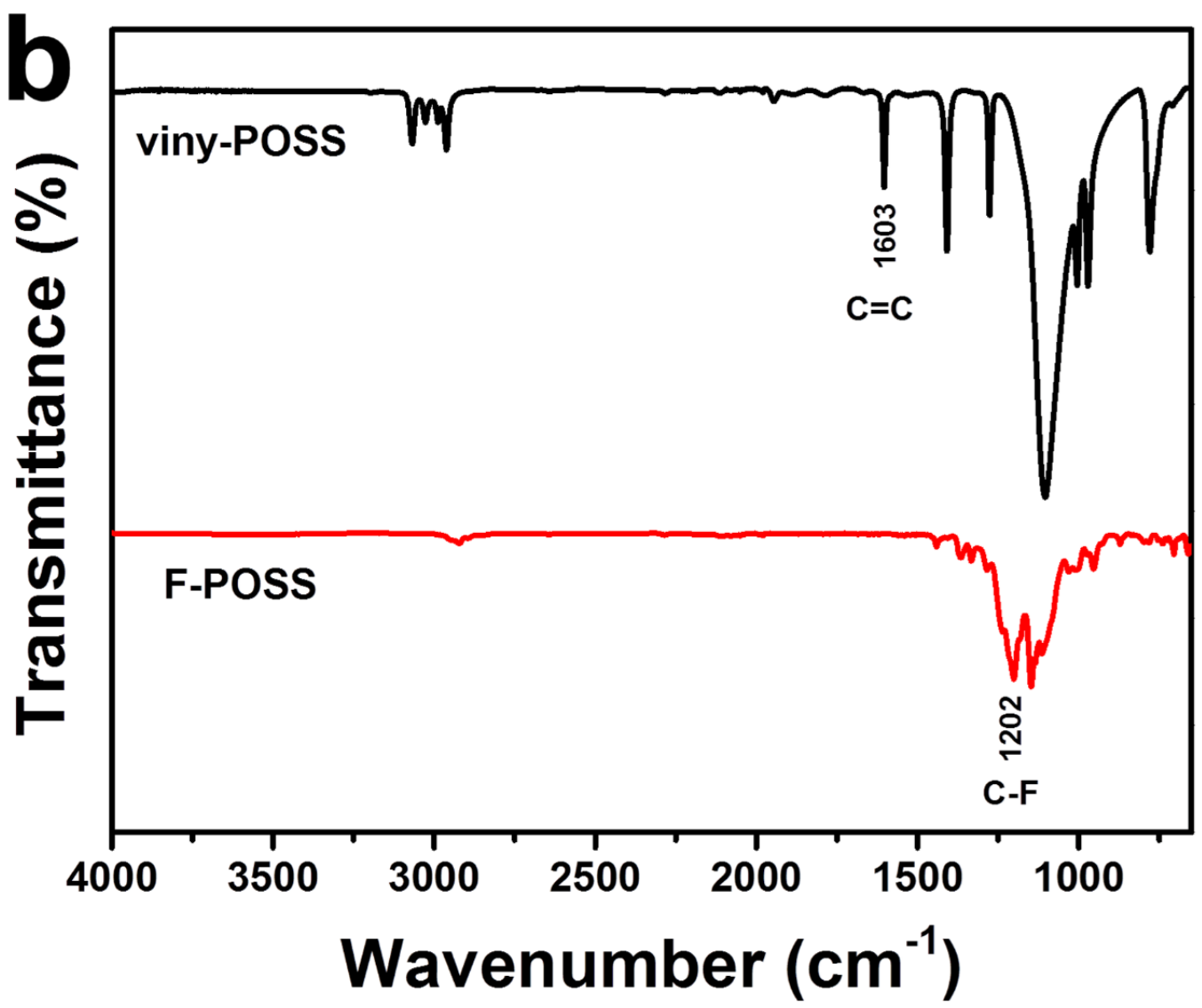

Figure S8. (a) Schematic of the synthesis of F-POSS. (b) Infrared spectra of viny-POSS and FPOSS.

It is clearly found that the stretching vibration of the $\mathrm{C}=\mathrm{C}$ at $1603 \mathrm{~cm}^{-1}$ nearly disappeared after reaction, whereas the stretching vibration of the C-F at $1202 \mathrm{~cm}^{-1}$ was detected. ${ }^{1,2}$ This indicates that the F-POSS has been successfully prepared. 
Table S1. Typical graphene-based and MXene-based MA materials and their MA properties in Xband.

\begin{tabular}{|c|c|c|c|c|c|}
\hline \multirow[b]{2}{*}{ Sample } & \multirow[b]{2}{*}{ Matrix } & \multicolumn{2}{|c|}{$R L<-10 \mathrm{~dB}(X-b a n d)$} & \multirow{2}{*}{$\begin{array}{l}\text { Minimum } \\
\text { RL (dB) }\end{array}$} & \multirow[b]{2}{*}{ Ref. } \\
\hline & & $\begin{array}{l}\text { Thickness } \\
\text { (mm) }\end{array}$ & $\begin{array}{l}\text { EAB } \\
\text { (GHz) }\end{array}$ & & \\
\hline $\mathrm{RGO} / \mathrm{ZnO}$ hollow spheres & Wax & 2.2 & 3.3 & -45.1 & $3(2014)$ \\
\hline $\mathrm{RGO} / \mathrm{MnFe}_{2} \mathrm{O}_{4}$ & PVDF & 3.0 & 4.2 & -29.0 & $4(2014)$ \\
\hline $\mathrm{RGO} / \mathrm{MoS}_{2}$ & Wax & 2.5 & $\sim 3.1$ & $\sim-25.0$ & $5(2015)$ \\
\hline CF@G@PPy aerogel & Wax & 2.5 & $\sim 3.4$ & $\sim-25.0$ & $6(2015)$ \\
\hline RGO foam & Wax & 10.0 & 4.2 & -27.0 & $7(2016)$ \\
\hline $\mathrm{Ti}_{3} \mathrm{C}_{2} \mathrm{~T}_{\mathrm{x}}$ powder & Wax & 1.8 & 2.8 & -30.0 & $8(2016)$ \\
\hline $\mathrm{RGO} / \mathrm{ZnOnws}$ foam & PDMS & 4.8 & 4.2 & -27.8 & $9(2017)$ \\
\hline $\mathrm{RGO} / \mathrm{SiCnws}$ foam & PDMS & 3.0 & 4.2 & -19.6 & $10(2017)$ \\
\hline Nitrogen-doped graphene $/ \mathrm{Ti}_{3} \mathrm{C}_{2} \mathrm{~T}_{\mathrm{x}}$ & Epoxy & 1.4 & 1.5 & $\sim-20.0$ & $11(2017)$ \\
\hline Edge-rich graphene & $\mathrm{Si}_{3} \mathrm{~N}_{4}$ & 3.75 & 4.2 & -26.7 & $12(2018)$ \\
\hline $\mathrm{RGO} / \mathrm{Ti}_{3} \mathrm{C}_{2} \mathrm{~T}_{\mathrm{x}}$ foam & None & 3.2 & 4.2 & -23.0 & $13(2018)$ \\
\hline $\mathrm{Ti}_{3} \mathrm{C}_{2} \mathrm{~T}_{\mathrm{X}} / \mathrm{SiCnws}$ foam & None & 2.6 & 3.2 & -14.7 & $14(2018)$ \\
\hline $\mathrm{Ti}_{3} \mathrm{C}_{2} \mathrm{~T}_{\mathrm{x}} / \mathrm{PI}$ aerogel & None & 3.0 & 3.7 & -45.4 & $15(2018)$ \\
\hline $\mathrm{Ti}_{3} \mathrm{C}_{2} \mathrm{~T}_{\mathrm{x}} /$ cellulose aerogel & Wax & 2.5 & 2.8 & -43.4 & $16(2018)$ \\
\hline $\mathrm{Ti}_{3} \mathrm{C}_{2} \mathrm{~T}_{\mathrm{x}} /$ nano-carbon-spheres & Wax & 2.2 & $\sim 2.9$ & -39.8 & $17(2018)$ \\
\hline $\mathrm{Ti}_{3} \mathrm{C}_{2} \mathrm{~T}_{\mathrm{x}} / \mathrm{FeCo}$ & Wax & 1.6 & 3.2 & -17.9 & $18(2019)$ \\
\hline $\mathrm{Ti}_{3} \mathrm{C}_{2} \mathrm{~T}_{\mathrm{x}} / \mathrm{CoFe}$ & Wax & 1.7 & $\sim 2.1$ & -34.9 & 19 (2019) \\
\hline $\mathrm{Ti}_{2} \mathrm{CT}_{\mathrm{X}} / \mathrm{PVA}$ foam & None & 3.4 & 4.2 & -18.7 & 20 (2019) \\
\hline $\mathrm{Ti}_{3} \mathrm{C}_{2} \mathrm{~T}_{\mathrm{x}} / \mathrm{Ni}$ chain & Wax & 1.75 & $\sim 1.7$ & -49.9 & $21(2019)$ \\
\hline $\mathrm{Ti}_{3} \mathrm{C}_{2} \mathrm{~T}_{\mathrm{x}} / \mathrm{Ni}-40$ & Wax & 2.5 & 4.2 & $\sim-32.0$ & $22(2019)$ \\
\hline $\mathrm{Ti}_{3} \mathrm{C}_{2} \mathrm{~T}_{\mathrm{x}} / \mathrm{TiO}_{2}$ & Wax & 2.0 & 2.8 & -19.8 & $23(2020)$ \\
\hline S-CF-MNZ-3 & $\begin{array}{l}\text { Cotton } \\
\text { fabric }\end{array}$ & 2.2 & 4.2 & -25.5 & $\begin{array}{l}\text { This } \\
\text { work }\end{array}$ \\
\hline
\end{tabular}




\begin{tabular}{|c|c|c|c|c|c|}
\hline S-CF-MNZ-6 & $\begin{array}{l}\text { Cotton } \\
\text { fabric }\end{array}$ & 2.4 & 4.2 & -32.4 & $\begin{array}{c}\text { This } \\
\text { work }\end{array}$ \\
\hline S-CF-MNZ-9 & $\begin{array}{l}\text { Cotton } \\
\text { fabric }\end{array}$ & 2.6 & 4.2 & -35.1 & $\begin{array}{l}\text { This } \\
\text { work }\end{array}$ \\
\hline
\end{tabular}

Table S2. The contents of active materials in fabrics.

\begin{tabular}{cccc}
\hline & MXene (wt\%) & Ni chain (wt\%) & ZnO array (wt\%) \\
\hline S-CF-MNZ-3 & 3.26 & 0.42 & 10.44 \\
S-CF-MNZ-6 & 3.27 & 0.75 & 9.67 \\
S-CF-MNZ-9 & 3.29 & 1.11 & 8.99 \\
\hline
\end{tabular}

\section{REFERENCES}

1. Chen, S.; Li, X.; Li, Y.; Sun, J. Intumescent Flame-Retardant and Self-Healing Superhydrophobic Coatings on Cotton Fabric. ACS Nano 2015, 9, 4070-4076.

2. Chen, D.; Sun, W.; Qian, C.; Reyes, L.; Wong, A.; Dong, Y.; Jia, J.; Chen, K.; Ozin, G. Porous NIR Photoluminescent Silicon Nanocrystals-POSS Composites. Adv. Funct. Mater. 2016, $26,5102-5110$.

3. Han, M.; Yin, X.; Kong, L.; Li, M.; Duan, W.; Zhang, L.; Cheng, L. Graphene-Wrapped ZnO Hollow Spheres with Enhanced Electromagnetic Wave Absorption Properties. J. Mater. Chem. A 2014, 2, 16403-16409.

4. Zhang, X. J.; Wang, G. S.; Cao, W. Q.; Wei, Y. Z.; Liang, J. F.; Guo, L.; Cao, M. S. Enhanced Microwave Absorption Property of Reduced Graphene Oxide (RGO)- $\mathrm{MnFe}_{2} \mathrm{O}_{4}$ Nanocomposites and Polyvinylidene Fluoride. ACS Appl. Mater. Interfaces 2014, 6, 7471-7478. 
5. Wang, Y.; Chen, D.; Yin, X.; Xu, P.; Wu, F.; He, M. Hybrid of $\mathrm{MoS}_{2}$ and Reduced Graphene Oxide: A Lightweight and Broadband Electromagnetic Wave Absorber. ACS Appl. Mater. Interfaces 2015, 7, 26226-26234.

6. Wang, C.; Ding, Y.; Yuan, Y.; He, X.; Wu, S.; Hu, S.; Zou, M.; Zhao, W.; Yang, L.; Cao, A.; Li, Y. Graphene Aerogel Composites Derived from Recycled Cigarette Filters for Electromagnetic Wave Absorption. J. Mater. Chem. C 2015, 3, 11893-11901.

7. Zhang, Y.; Huang, Y.; Chen, H.; Huang, Z.; Yang, Y.; Xiao, P.; Zhou, Y.; Chen, Y. Composition and Structure Control of Ultralight Graphene Foam for High-Performance Microwave Absorption. Carbon 2016, 105, 438-447.

8. Han, M.; Yin, X.; Wu, H.; Hou, Z.; Song, C.; Li, X.; Zhang, L.; Cheng, L. Ti ${ }_{3} \mathrm{C}_{2}$ MXenes with Modified Surface for High-Performance Electromagnetic Absorption and Shielding in the XBand. ACS Appl. Mater. Interfaces 2016, 8, 21011-21019.

9. Song, C.; Yin, X.; Han, M.; Li, X.; Hou, Z.; Zhang, L.; Cheng, L. Three-Dimensional Reduced Graphene Oxide Foam Modified with $\mathrm{ZnO}$ Nanowires for Enhanced Microwave Absorption Properties. Carbon 2017, 116, 50-58.

10. Han, M.; Yin, X.; Hou, Z.; Song, C.; Li, X.; Zhang, L.; Cheng, L. Flexible and Thermostable Graphene/SiC Nanowire Foam Composites with Tunable Electromagnetic Wave Absorption Properties. ACS Appl. Mater. Interfaces 2017, 9, 11803-11810.

11. Qing, Y.; Nan, H.; Luo, F.; Zhou, W. Nitrogen-Doped Graphene and Titanium Carbide Nanosheet Synergistically Reinforced Epoxy Composites as High-Performance Microwave Absorbers. RSC Adv. 2017, 7, 27755-27761. 
12. Ye, F.; Song, Q.; Zhang, Z.; Li, W.; Zhang, S.; Yin, X.; Zhou, Y.; Tao, H.; Liu, Y.; Cheng, L.; Zhang, L.; Li, H. Direct Growth of Edge-Rich Graphene with Tunable Dielectric Properties in Porous $\mathrm{Si}_{3} \mathrm{~N}_{4}$ Ceramic for Broadband High-Performance Microwave Absorption. Adv. Funct. Mater. 2018, 28, 1707205.

13. Li, X.; Yin, X.; Song, C.; Han, M.; Xu, H.; Duan, W.; Cheng, L.; Zhang, L. Self-Assembly Core-Shell Graphene-Bridged Hollow MXenes Spheres 3D Foam with Ultrahigh Specific EM Absorption Performance. Adv. Funct. Mater. 2018, 28, 1803938.

14. Li, X.; Yin, X.; Xu, H.; Han, M.; Li, M.; Liang, S.; Cheng, L.; Zhang, L. Ultralight MXeneCoated, Interconnected SiCnws Three-Dimensional Lamellar Foams for Efficient Microwave Absorption in the X-Band. ACS Appl. Mater. Interfaces 2018, 10, 34524-34533.

15. Liu, J.; Zhang, H. B.; Xie, X.; Yang, R.; Liu, Z.; Liu, Y.; Yu, Z. Z. Multifunctional, Superelastic, and Lightweight MXene/Polyimide Aerogels. Small 2018, 14, 1802479.

16. Jiang, Y.; Xie, X.; Chen, Y.; Liu, Y.; Yang, R.; Sui, G. Hierarchically Structured Cellulose Aerogels with Interconnected MXene Networks and Their Enhanced Microwave Absorption Properties. J. Mater. Chem. C 2018, 6, 8679-8687.

17. Dai, B.; Zhao, B.; Xie, X.; Su, T.; Fan, B.; Zhang, R.; Yang, R. Novel Two-Dimensional $\mathrm{Ti}_{3} \mathrm{C}_{2} \mathrm{~T}_{\mathrm{x}}$ MXenes/Nano-Carbon Sphere Hybrids for High-Performance Microwave Absorption. $J$. Mater. Chem. C 2018, 6, 5690-5697.

18. He, J.; Shan, D.; Yan, S.; Luo, H.; Cao, C.; Peng, Y. Magnetic FeCo NanoparticlesDecorated $\mathrm{Ti}_{3} \mathrm{C}_{2}$ MXene with Enhanced Microwave Absorption Performance. J. Magn. Magn. Mater. 2019, 492, 165639. 
19. Zhou, C.; Wang, X.; Luo, H.; Deng, L.; Wang, S.; Wei, S.; Zheng, Y.; Jia, Q.; Liu, J. Interfacial Design of Sandwich-Like CoFe@ $\mathrm{Ti}_{3} \mathrm{C}_{2} \mathrm{~T}_{\mathrm{x}}$ Composites as High Efficient Microwave Absorption Materials. Appl. Surf. Sci. 2019, 494, 540-550.

20. Xu, H.; Yin, X.; Li, X.; Li, M.; Liang, S.; Zhang, L.; Cheng, L. Lightweight $\mathrm{Ti}_{2} \mathrm{CT}_{\mathrm{x}}$ MXene/Poly(vinyl Alcohol) Composite Foams for Electromagnetic Wave Shielding with Absorption-Dominated Feature. ACS Appl. Mater. Interfaces 2019, 11, 10198-10207.

21. Liang, L.; Han, G.; Li, Y.; Zhao, B.; Zhou, B.; Feng, Y.; Ma, J.; Wang, Y.; Zhang, R.; Liu, C. Promising $\mathrm{Ti}_{3} \mathrm{C}_{2} \mathrm{~T}_{\mathrm{x}}$ MXene/Ni Chain Hybrid with Excellent Electromagnetic Wave Absorption and Shielding Capacity. ACS Appl. Mater. Interfaces 2019, 11, 25399-25409.

22. Li, X.; You, W.; Wang, L.; Liu, J.; Wu, Z.; Pei, K.; Li, Y.; Che, R. Self-AssemblyMagnetized MXene Avoid Dual-Agglomeration with Enhanced Interfaces for Strong Microwave Absorption through a Tunable Electromagnetic Property. ACS Appl. Mater. Interfaces 2019, 11, $44536-44544$.

23. Fan, B.; Shang, S.; Dai, B.; Zhao, B.; Li, N.; Li, M.; Zhang, L.; Zhang, R.; Marken, F. 2DLayered $\mathrm{Ti}_{3} \mathrm{C}_{2} / \mathrm{TiO}_{2}$ Hybrids Derived from $\mathrm{Ti}_{3} \mathrm{C}_{2}$ MXenes for Enhanced Electromagnetic Wave Absorption. Ceram. Int. 2020, 46, 17085-17092. 